\title{
Glass micro-cutting by Spark Assisted Chemical Engraving
}

\author{
Lucas A. Hof, Rolf Wüthrich \\ Department of Mechanical \& Industrial Engineering \\ Concordia University \\ Montreal, Canada \\ lucas.hof@concordia.ca, rolf.wuthrich@concordia.ca
}

\begin{abstract}
Glass remains a difficult to machine material. Its brittleness, chemical resistance and relatively high thermal conductivity challenges available technologies. An interesting approach is to the use of hybrid technologies such as Spark Assisted Chemical Engraving (SACE) which is a thermochemical process. Micro-drilling and micro-milling were intensively characterised and developed. SACE can however as well be used to micro-cut glass. This aspect was so far never discussed in the literature and no systematic data about its performance are available. As a first step, basic parameters such as depth of cut and tool feed rate must be determined. This communication aims to report systematic experiments on micro-cutting glass by SACE technology and demonstrates how using available data from SACE microdrilling the maximal depth-of-cut and tool feed can be determined. Relations for depth-of-cut and tool feedrate are proposed to use as guidance for glass micro-cutting.
\end{abstract}

Keywords - micro-machining; glass; spark assisted chemical engraving; micro-cutting; advanced manufacturing

\section{INTRODUCTION}

The application of glass science to the improvement of industrial tools occurred only in the past century, with a few exceptions. Glass has been employed in many forms to fabricate glazing and containers for centuries while it is now entering new applications that are appearing in micro and even nanotechnology like fibers, displays and Micro-ElectroMechanical-System (MEMS) devices [1]. Many qualities make glass attractive since it is transparent, chemically inert, environmentally friendly and its mechanical strength and thermal properties. In fact, no other materials being massproduced have shown such qualities over so many centuries. Nowadays glass offers recycling opportunities and allows for tailoring new and dedicated applications. Moreover, glass is radio frequency (RF) transparent, making it an excellent material for sensor and energy transmission devices. Another advantage of using glass in microfluidic MEMS devices [2] is its relatively high heat resistance, which makes these devices suitable for high temperature microfluidic systems [3] and sterilization by autoclaving.
However, glass remains a difficult to machine material. Its brittleness, chemical resistance and relatively high thermal conductivity challenges available technologies. An interesting approach is to the use of hybrid technologies such as Spark Assisted Chemical Engraving (SACE) [4] which is a thermochemical process (Fig. 1).

In SACE process, a voltage is applied between tool- and counter-electrode dipped in an alkaline solution - typical $\mathrm{NaOH}$ or $\mathrm{KOH}$ (Fig. 1). At high voltages (around $30 \mathrm{~V}$ ), the bubbles evolving around the tool electrode coalesce into a gas film and discharges occur from the tool to the electrolyte through it. Glass machining becomes possible due to thermally promoted etching (breaking of the Si-O-Si bond) [4].
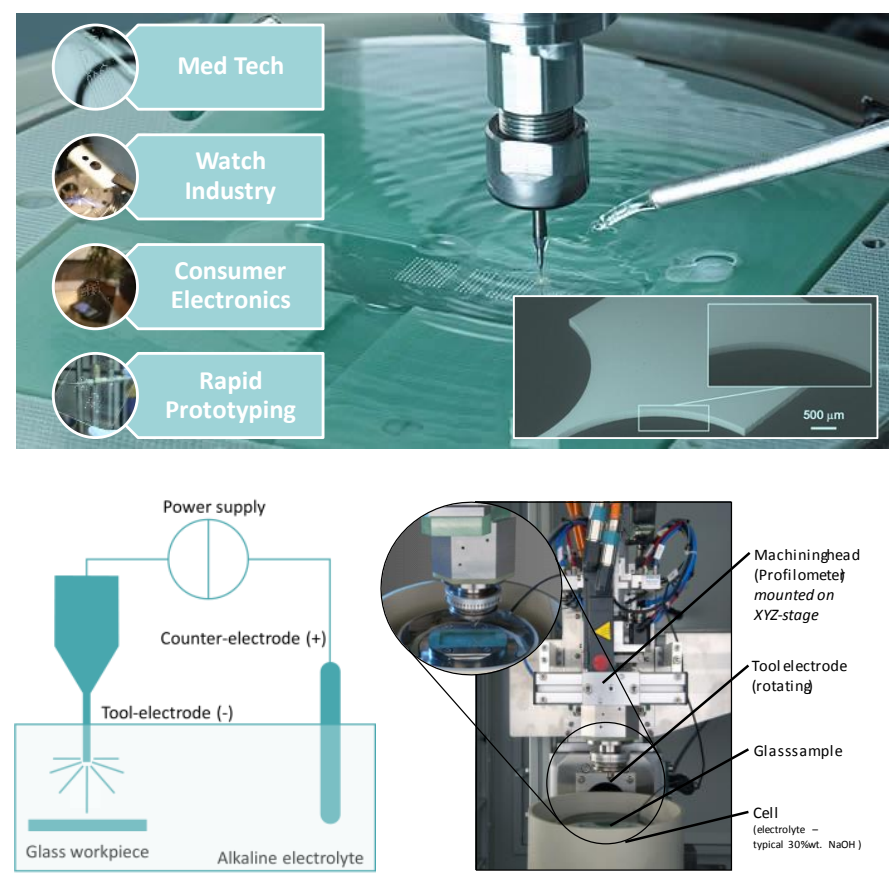

Figure 1. Above left: Overview of targeted applications/markets for SACE glass machining. Above right: SACE machined micro-hinge (scale bar $=500$ $\mu \mathrm{m}$ - width of 'hinge' $=30 \mu \mathrm{m}$ - thickness glass $=100 \mu \mathrm{m})$. Below left: Principles of SACE machining. Below right: SACE machining head. 
Developed since the sixties in last century in academia [5], SACE found recently its entrance in the industrial world. Micro-drilling and micro-milling were intensively characterised and developed. The introduction of pulsed voltage machining did made a further significant step forward in term of quality of machining. SACE can however as well be used to micro-cut glass. This aspect was so far never discussed in the literature and no systematic data about its performance are available. As a first step, basic parameters such as depth of cut and tool feed rate must be determined. This is the aim of the present communication which reports systematic experiments on micro-cutting glass by SACE and demonstrates how using available data from SACE micro-drilling maximal depth-of-cut and tool feed can be determined.

\section{MATERIALS AND METHODS}

Micro-cutting of pyrex glass (microscope slides) was performed on a Posalux FP1-SACE machine (Fig. 2). The machine incorporates a force sensor in the machining head that allows online measurement of the axial force (down to $1 \mathrm{mN}$ ) exerted on the tool-electrode. As electrolyte 30wt\% Potassium Hydroxide $(\mathrm{KOH})$, prepared from de-ionised water, was used. Low viscous electrolyte is chosen to reduce the chance that flushing with fresh electrolyte at the machining zone becomes the limiting factor for machining/cutting.

Cylindrical tool electrodes, made of tungsten carbide, 100 $\mu \mathrm{m}$ diameter with a bevel of $45^{\circ}$ where used. The spindle rotated at $1000 \mathrm{rpm}$. Cuts were performed over an equivalent length of at least two minutes of micro-cutting, adapted at the operating cutting speed.

After cutting, images with an optical microscope (Keyence VHX 5000) were acquired in order to evaluate precisely the realized depth-of-cut and to evaluate the cut qualitatively.



Figure 2. Posalux FP1-SACE machine (modular system)

\section{THEORY}

Material removal rate in SACE machining is limited, in case flushing of the machining zone happens easily, by heat propagation in the glass workpiece [4]. In the case of microcutting, electrolyte can access freely to the machining zone by appropriate flushing, using side tubes for electrolyte supply in the machining cell (Fig. 1). Further machined material is removed readily from it through the cut. Consequently, as a first approximation, one can discuss the relation between the axial depth of cut $p$ and horizontal feed rate $F$ (see Fig. 3 for a schematic) in terms of time needed for the heat to propagate inside the workpiece: one has to give enough time to the workpiece to heat up while one moves the tool.

Consider a tool of radius $r$. In the time interval

$$
t_{F}=\frac{2 r}{F}
$$

the tool will move over its own size. If heat propagates at the rate $h_{r}$ inside the workpiece, then the time $t_{h}$ needed to heat a distance $p$ is given by

$$
t_{h}=\frac{p}{h_{r}}
$$

If heat propagation is the rate limiting process, the tool cannot move faster than the feed rate $F$ at an axial depth of cut $p$ such that ( $t_{F}$ and $t_{h}$ must be of the same order of magnitude):

$$
F \cdot p=2 r \cdot h_{r}
$$

Equation (3) shows that, under the stated hypothesis that machining is only limited by heat propagation, the maximal value of the product $F \cdot p$ is, for a given tool and workpiece, a constant value. Note that, as the time $t$ needed to cut a path of length $L$ and depth $d$ is given by

$$
t=L \cdot d \frac{1}{F \cdot p}
$$

in SACE cutting, the time needed to perform a given cut is essentially constant regardless of the values of $F$ and $p$ chosen.

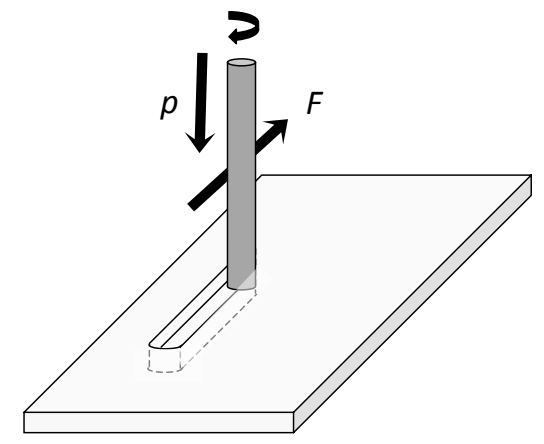

Figure 3. Schematic representation of micro-cuting by SACE process with a rotating tool-electrode (radius $r$ ) moving horizontally with feedrate $F$ at an axial depth of cut $p$. 
The heat propagation rate $h_{r}$ is function of the geometry of the problem and the energy put into the system (i.e. energy produced by the spark activity around the tool electrode). Estimations of the heat propagation rate in case of drilling for cylindrical tools are reported in the literature [4] and can be used as a first input.

Explicit calculation of the product $F \cdot p$ using equation (5.23) from [4] is found to be:

$$
F \cdot p=4 a \cdot \frac{\kappa-1}{\kappa}\left[1-\exp \left(-\pi\left(\frac{\kappa-1}{\kappa}\right)^{2}\right)\right]
$$

with $a$ the thermal diffusity of the workpiece $\left(a \sim 6 \cdot 10^{-7} \mathrm{~m}^{2} / \mathrm{s}\right.$ for Pyrex ${ }^{\circledR}$ glass). Relations between the dimensionless number $\kappa$ and voltage $U$ are empirically derived in section 5.2 of [4]:

$$
\kappa=0.018 \cdot U+0.615
$$

Equation (5) and (6) allows to establish a direct relationship between the machining voltage $U$ and the product $F \cdot p$ which can be used to define the material removal rate (MRR) when multiplying with the tool diameter.

\section{RESULTS AND DISCUSSION}

To determine experimentally the maximal depth-of-cut $\mathrm{p}$ at a given tool feed rate $\mathrm{F}$ a series of cuttings of $0.4 \mathrm{~mm}$ thick glass slides $\left(\right.$ Pyrex $^{\circledR}$ ) were performed. The cut was considered to be successful if

a) The tool didn't break, and

b) The measured depth of the cut was equal or higher than the imposed depth of cut during machining.

From a practical point of view, thanks to the force sensor inside the tool-electrode holder, it was possible to detect none successful cuts due to an increased force acting on the tool (a few $\mathrm{mN})$. Pulsed voltage with $2.5 \mathrm{~ms}$ pulse high time $\left(t_{\text {high }}\right)$ and $0.1 \mathrm{~ms}$ pulse low time $\left(t_{\text {low }}\right)$ was used. In such conditions the gas film shows high stability and very few bubbles are observed around the tool-electrode. Cutting experiments were performed at three pulsed machining voltages (high, intermediate and low) as denoted in table 1 .

The average input voltage $U_{\text {avg }}$ can be calculated according:

$$
U_{\text {avg }}=\left(t_{\text {high }}-t_{\text {low }}\right) /\left(t_{\text {high }}+t_{\text {low }}\right) \Delta U_{\text {pulse }}+U_{\text {lowlevel }}
$$

TABLE I. SACE MACHINING SETTINGS

\begin{tabular}{lcccc}
\hline \multicolumn{4}{c}{ Pulsed Voltage Input } \\
\hline & High Level & Low Level & Period & Duty Cycle \\
\hline Low & $30 \mathrm{~V}$ & $17.5 \mathrm{~V}$ & $2.6 \mathrm{~ms}$ & $96.15 \%$ \\
Intermediate & $33 \mathrm{~V}$ & $17.5 \mathrm{~V}$ & $2.6 \mathrm{~ms}$ & $96.15 \%$ \\
High & $35 \mathrm{~V}$ & $17.5 \mathrm{~V}$ & $2.6 \mathrm{~ms}$ & $96.15 \%$ \\
\hline
\end{tabular}

TABLE II. HEAT PROPAGATION IN GLASS WORKPIECE

\begin{tabular}{ll}
\hline Machining voltage $[\mathbf{V}]$ & F·p $\left[\mathrm{mm}^{2} / \mathbf{m i n}\right]$ \\
\hline 30 & 1.0 \\
33 & 1.6 \\
35 & 2.2 \\
\hline
\end{tabular}

Using these machining voltages (table 1 and equation (7)) as input in equations (5) and (6) we estimate the values for $F$. $p$ as outlined in table 2 .

As shown in Fig. 4, the maximal depth of cut for a given tool feed follows equation (3). The dashed lines were obtained by least square fits.

The quality of the cut is assessed by optical microscopy for the different machining voltages (low - intermediate - high voltage) and at varying feed rate and depth-of-cut (Fig. 5).

It can be clearly observed that quality of the glass cut generally increases when the machining voltage reduces (indents of $\sim 75 \mu \mathrm{m}-60 \mu \mathrm{m}-50 \mu \mathrm{m}$ for respectively $35 \mathrm{~V}-$ $33 \mathrm{~V}-30 \mathrm{~V}$ at maximum cutting depth for each voltage and feedrate $=5 \mathrm{~mm} / \mathrm{min}$.)). Decreasing the depth of cut results generally also in higher machining quality. In the extremes, when machining at same feedrate $-F=40 \mathrm{~mm} / \mathrm{min}$ - and low depth of cut, the machining quality is around the same for all applied machining voltages.

Reasonable quality of cut in glass by SACE technology can be obtained using the derived tool feed vs. depth of cut relations. It should be noted that this model is not optimized for quality of cut surface and other strategies, such as 'SACE polishing' by approaching the surface with small lateral increments of the tool-electrode at very low voltage, can be followed to achieve surface qualities in the order of a few microns.

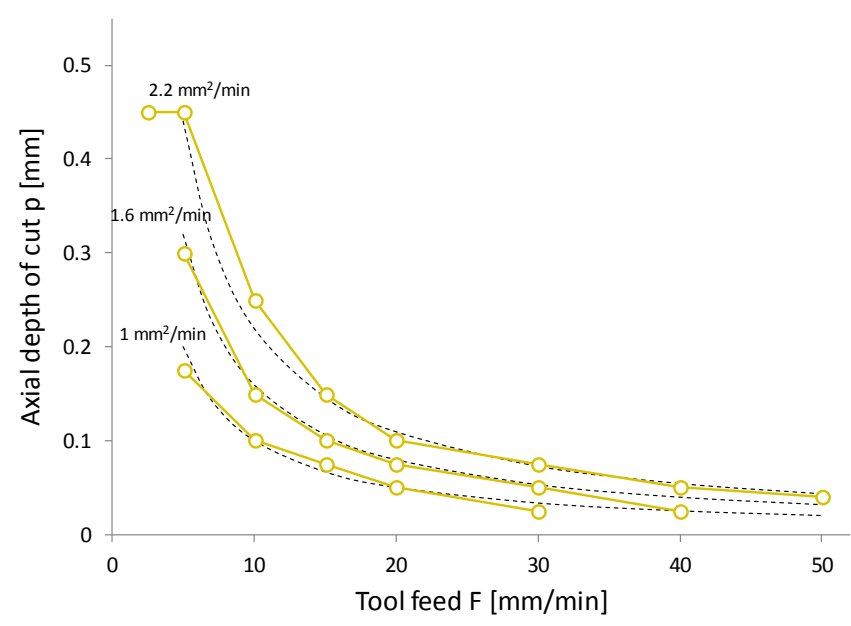

Figure 4. Yellow: Empirical values for maximal depth-of-cut $p[\mathrm{~mm}]$ versus tool feedrate $F[\mathrm{~mm} / \mathrm{min}]$ for tungsten carbide $100 \mu \mathrm{m}$ diameter tools. Grey dashed lines: Theoretical model values. 
Machining voltage $-35 \mathrm{~V}: 17.5 \mathrm{~V} / 2.5 \mathrm{~ms}: 0.1 \mathrm{~ms}$

Glass thickness: $400 \mu \mathrm{m}$

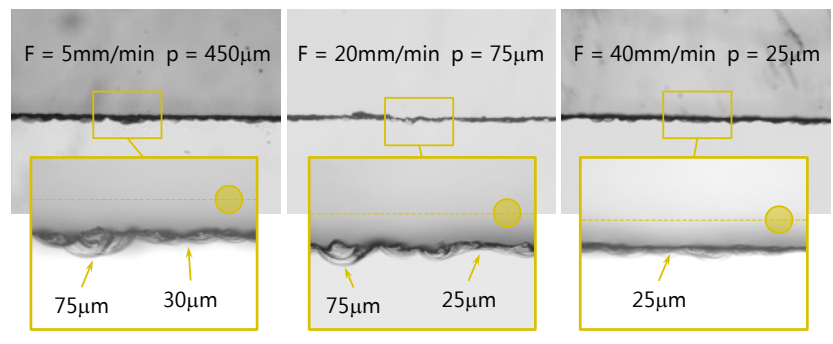

Machining voltage $-33 \mathrm{~V}: 17.5 \mathrm{~V} / 2.5 \mathrm{~ms}: 0.1 \mathrm{~ms}$

Glass thickness: $400 \mu \mathrm{m}$

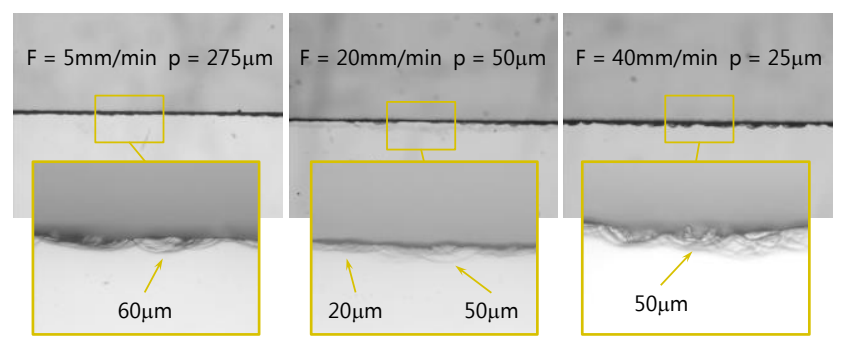

Machining voltage $-30 \mathrm{~V}: 17.5 \mathrm{~V} / 2.5 \mathrm{~ms}: 0.1 \mathrm{~ms}$

Glass thickness: $400 \mu \mathrm{m}$

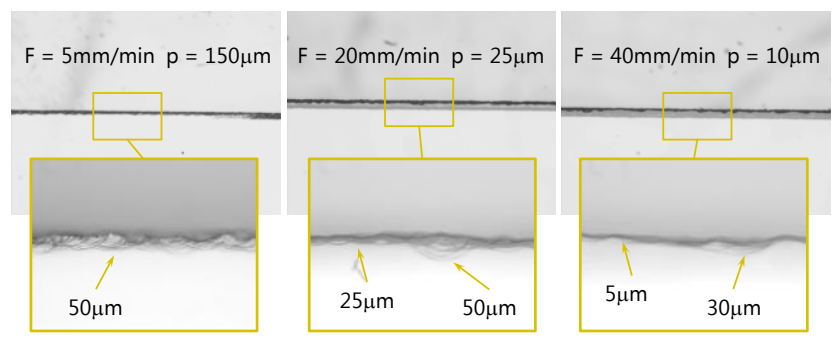

Figure 5. Microscopy (Keyence VHX 5000) analysis of cut quality at different machining voltage, feedrate and depth-of-cut.

\section{CONCLUSIONS}

- Cutting parameters for tungsten carbide $100 \mu \mathrm{m}$ diameter cylindrical tools (with $45^{\circ}$ bevel) were determined;

- A simple model relating the lateral tool feedrate $F$ to the maximum depth-of-cut $p$ based on the assumption that machining is only limited by heat tranfer in the workpiece was presented and matches well the measured data;

- Cut quality was evaluated by optical micro-graphs for different machining voltages (high, medium and low voltage).

The developed model allows to predict the maximum depth of cut for a given tool feedrate, which eliminates timeconsuming 'trial runs' before machining to determine the best cutting parameters. Hence, this study contributes to further optimization of SACE technology for industrial use.

\section{ACKNOWLEDGMENT}

This work was supported by the Natural Sciences and Engineering Research Council of Canada (NSERC). L.A.H. would like to thank the Quebec MEESR for the 'bourse d'excellence pour étudiants étrangers' (Doctoral students V1) and acknowledge Posalux SA for the fruitful discussions and work performed on their FP1-SACE machine.

\section{REFERENCES}

[1] E. Le Bourhis, Glass, Mechanics and Technology. Wiley-VCH, 2014.

[2] G. M. Whitesides, "The origins and the future of microfluidics.," Nature, vol. 442, no. 7101, pp. 368-373, 2006.

[3] D. Sinton, "Energy: the microfluidic frontier.," Lab Chip, vol. 14, no. 17, 2014.

[4] R. Wüthrich and J. D. Abou Ziki, Micromachining Using Electrochemical Discharge Phenomenon. Elsevier, 2015.

[5] H. Kurafuji and K. Suda "Electrical discharge drilling of glass" Annals of the CIRP, 16, pp. $415-419,1968$. 